\title{
Las habilidades sociales desde las experiencias y representaciones de la enseñanza en la modalidad virtual
}

\author{
Social skills from the experiences and representations of teaching in the virtual
}

Habilidades sociais a partir das experiências e representações do ensino na modalidade virtual

\author{
Cecilia Elizalde Cordero ${ }^{1}$ \\ cecielizaldecor@hotmail.com \\ https://orcid.org/0000-0000-3822-1725 \\ Liliana Baque Pibaque ${ }^{2}$ \\ liliana.baquep@ug.edu.ec \\ https://orcid.org/0000-0002-3720-0597
}

\author{
Miriam Calle Cobos ${ }^{2}$ \\ miriam.callec@ug.edu.ec \\ https://orcid.org/0000-0002-6699-5835 \\ Rosy Cañizares Hermoza ${ }^{3}$ \\ rcañizares@mariscalsucre.edu.ec \\ https://orcid.org/0000-0001-6976-4058
}

${ }^{1}$ Universidad César Vallejo, Piura-Perú

${ }^{2}$ Universidad de Guayaquil, Guayaquil-Ecuador

${ }^{3}$ Unidad Educativa Mariscal Sucre, Guayaquil-Ecuador

Artículo recibido en junio 2021, revisado en julio 2021, arbitrado en agosto 2021 y publicado en septiembre 2021

\section{RESUMEN}

La educación en casa como modalidad educativa por motivo de coronavirus convirtió a los padres en maestros desde sus hogares, bajo parámetros de zozobra, poca tolerancia y estrés; nadie estaba preparado para iniciar la virtualidad. El objetivo de investigación fue analizar las experiencias y representaciones de la enseñanza desde la modalidad virtual. El estudio se realizó desde un enfoque cualitativo, se orientó hacia un diseño de campo, que permitió observar y recolectar información. Resultó que la propuesta del desarrollo de las habilidades sociales permitió establecer, consolidar y regular las interacciones con padres y estudiantes especialmente en la modalidad virtual con el fin de regular dificultades y sentimientos ansiosos en situaciones sociales, así como los valores y metas que regirán el proceso de cambio. Se concluyó que la problemática más grande que los docentes con déficits en la adquisición de habilidades sociales es la barrera socioemocional.

Palabras clave: Habilidades sociales; Experiencias y representaciones; Docentes, Modalidad virtual

\section{ABSTRACT}

Home schooling as an educational modality due to coronavirus turned parents into teachers from their homes, under parameters of anxiety, little tolerance and stress; no one was prepared to initiate virtuality. The research objective was to analyze the experiences and representations of teaching from the virtual modality. The study was carried out from a qualitative approach, it was oriented towards a field design, which allowed observing and collecting information. It turned out that the proposal for the development of social skills allowed to establish, consolidate and regulate interactions with parents and students, especially in the virtual mode, in order to regulate difficulties and anxious feelings in social situations, as well as the values and goals that will govern the process. exchange. It was concluded that the biggest problem for teachers with deficits in the acquisition of social skills is the socio-emotional barrier.

Key words: Social skills; Experiences and representations; Teachers, Virtual modality

\section{RESUMO}

A escolarização em casa como modalidade educacional devido ao coronavírus transformou os pais em professores de suas casas, sob parâmetros de ansiedade, pouca tolerância e estresse; ninguém estava preparado para iniciar a virtualidade. $\mathrm{O}$ objetivo da pesquisa foi analisar as experiências e representações do ensino na modalidade virtual. O estudo foi realizado a partir de uma abordagem qualitativa, orientado para um delineamento de campo, o que permitiu observar e coletar informações. Constatou-se que a proposta de desenvolvimento de habilidades sociais permitiu estabelecer, consolidar e regular as interações com pais e alunos, principalmente na modalidade virtual, de forma a regular as dificuldades e sentimentos de ansiedade em situações sociais, bem como os valores. e objetivos que irão reger o processo. Concluiu-se que o maior problema para professores com déficit na aquisição de habilidades sociais é a barreira socioemocional.

Palavras chave: Habilidades sociais; Experiências e representações; Professores, Modalidade virtual. 


\section{INTRODUCCIÓN}

Para el presente estudio fue necesario partir de la definición de habilidad, es decir del desempeño de las personas en un área específica. Las habilidades se consideran como talentos innatos, naturales, que pueden ser aprendidos o perfeccionados, la misma implica el desarrollo y práctica de algún talento en potencia. Una vez que se tuvo una idea más precisa de lo que es habilidad, entonces de inmediato se procedió a construir una definición sobre habilidades sociales, estas se entienden como aquella destreza, capacidad o competencia hacia la interacción social, aquellas que involucran al trato con las demás personas, mediante la comunicación, participación, liderazgo y empatía.

Las habilidades sociales son un conjunto de conductas, pensamientos, emociones que permiten a las personas relacionarse con otras, se forman por la expresión de opiniones, sentimientos, son necesarias y se desarrollan desde los primeros meses de vida y están presentes durante ella, por lo que es imposible no prescindir de ellas en todo ambiente del cual forma parte, sea la familia, la comunidad, la escuela, por lo que resultan de vital importancia para la integración a un grupo de personas.

Es pertinente citar a Caballo (1991), pues tiene un enfoque plural de la temática:

las habilidades sociales (HHSS) son ese conjunto de conductas emitidas por una persona en el contexto interpersonal que le permiten expresar sus sentimientos, actitudes, deseos, opiniones o derechos de un modo adecuado a la situación, respetando esas conductas en los demás, logrando en general, de este modo, resolver los problemas inmediatos de la situación mientras reduce la probabilidad de futuros problemas (p.407).
$\mathrm{Al}$ respecto, los desarrollos de las habilidades sociales son de gran ayuda para todas las personas, por medio de ellas se logra pensar mejor, debido a que son una fuente de satisfacción personal. La falta de vínculo social genera estrés, malestar, momentos depresivos de ansiedad, miedo de comunicarse con los otros; lo que no ocurriría si se mantienen habilidades sociales positivas, ya que esta última otorga oportunidades de expresar amor, afecto, pedir con agrado, participar a gusto, establecer comunicación efectiva, generando de esta manera vínculos afectivos.

Para Gil y León (2019) las habilidades sociales permiten desempeñar las siguientes funciones: a) son reforzadores en situaciones de interacción social; b) mantienen o mejoran la relación interpersonal con otros individuos. Una buena relación y comunicación son puntos base para posteriores intervenciones con cualquier tipo de colectivo; c) impiden el bloqueo del reforzamiento social de las personas significativas para el sujeto; $\mathrm{d}$ ) disminuye el estrés y la ansiedad ante determinadas situaciones sociales y e) mantiene y mejora tanto la autoestima como el auto concepto.

Existen algunos tipos de habilidades sociales, entre ellas las básicas que tienen que ver con la escucha activa, el poder conversar, expresar y recibir estímulos positivos y negativos, habilidades avanzadas para pedir ayuda, seguir instrucciones, habilidades sociales que ayudan a controlar la agresión y socialización con otras personas.

Así, por ejemplo, cuando se interacciona con los padres desde el hogar, se puede relacionar de dos maneras: padres asertivos y padres agresivos. De los padres agresivos solo se aprenderán conductas no gratas, el grito y el golpe se convierten en acciones para conseguir algo. Al contrario, ocurre cuando se cuenta con padres asertivos, aquellos que son 
capaces de comunicarse con paciencia, respeto, tienen la disposición para explicar lo que está bueno o malo, de manera de hacerse entender sin ofender. Adicionalmente se expresan de una forma constructiva, se muestran seguros de sí mismo. Se estima que es lógico que esas conductas dadas por ellos serán imitadas, porque así lo aprendieron.

Es pertinente destacar al respecto lo que expresa Moscovici (1979), acerca de que las representaciones se fundamentan en la dialéctica que se instaura entre las interacciones cotidianas de los sujetos, su mundo de experiencias previas y las condiciones del entorno y sirven para orientarse en el contexto social.

Por su parte, también se suma la opinión acertada de Villarroel (1999) en relación a que "las representaciones sociales se cimientan en formas de conocimiento de tipo práctico que se exteriorizan en las sociedades contemporáneas de forma adyacentes a las experiencias de vida" (p. 6). Constituyéndose así en el origen de la teoría del conocimiento fundamentada en el pensamiento simbólico y la capacidad de representar una cosa mediante otra a través de un concepto simbolizan las relaciones sociales.

Se podría afirmar que es una característica particular del conocimiento, cuya función es la elaboración de los comportamientos y la comunicación entre los individuos. Para Vergara Quintero (2008). Las representaciones sociales agrupan informaciones, imágenes, opiniones, actitudes, ritos, técnicas, costumbres, modas, sentimientos, creencias, miedos, que nos permiten vivir en el entorno social. Es necesario considerar que los elementos constitutivos de una representación social surgen a partir de dos procesos: la objetivación y el anclaje. Adicionalmente es oportuno comprender que las experiencias y representaciones en la enseñanza se pueden presentar de muchas formas para clasificar las circunstancias de las situaciones que pasamos con otras personas.

Por otro lado, el desarrollo del aprendizaje en entornos virtuales de enseñanza aprendizaje son espacios educativos a distancia, constituyendo de vital importancia la formación y las actitudes que tenga el docente en torno al dominio tecnológico, en relación a las habilidades sociales estudiadas se encontró que en un alto grado de docentes que luego del acompañamiento brindado los demostraron a través del entorno virtual una conducta socialmente habilidosa en los aspectos conversacionales y de aceptación y oposición asertiva, basados en un trabajo colaborativo.

Se podría afirmar, siguiendo a Villasana y Dorrego (2017) y Cervera (2015) que las aplicaciones virtuales integradas, permiten crear un proceso flexible de enseñanza, pero quienes dirigen estos procesos necesitan de docente preparados y con grandes habilidades sociales para mantener conectados de forma significativa al aprendizaje que hoy en día se oferta.

Desde el aporte que otorgan las investigaciones previas para la ampliación del objeto de estudio, se considera propicio valorar las experiencias vivenciales durante el presente año donde la educación se convirtió en virtual, debido a que los estudiantes no podían tener acceso a las aulas, situación conocida por la presencia de la pandemia del Covid-19, que obligó a un aislamiento total en su comienzo y que el único medio de participación era precisamente a través de las diferentes plataformas virtuales al que podían tener acceso unos y otros no. Por diferentes motivos: falta de dispositivos tecnológicos, cobertura de internet al lugar de vivienda, desconocimiento de su uso, a pesar de los problemas presentados, los docentes han utilizado las estrategias más adecuadas para poder llegar a los 
estudiantes tratando de establecer una cultura de colaboración entre los docentes y estudiantes.

La labor del docente, desde la modalidad virtual, requirió inmediatamente de una preparación adecuada para su trabajo profesoral, de manera especial incentivando en cada uno de los involucrados en los procesos de enseñanza aprendizaje aspectos como: la responsabilidad individual y el asumir roles que los lleven a ser personas más eficientes. La presencia de las tecnologías de la comunicación en el ámbito educativo han sido motivo del crecimiento de la diferentes entornos virtuales que están al alcance de docentes, estudiantes, público en general, ofreciendo nuevos espacios para la enseñanza de una manera diferente ya no presencial, sino a través de un dispositivo tecnológico, es de suponer que al comienzo quien no estuvo preparado para este cambio, las cosas no les resultó tan fácil, en la actualidad ya son utilizados por diferentes instituciones educativos como apoyo al proceso educativo.

Así, el objetivo del presente estudio fue determinar el nivel de conocimientos que tienen los docentes para manejar las habilidades sociales en la modalidad virtual desde las experiencias y representaciones docentes. Se justifica la investigación debido a que el bienestar social y metodológico de los docentes es muy importante porque impacta de forma positiva en el proceso de aprendizaje de los estudiantes. El facilitarles espacios de diálogo donde expongan las inquietudes y necesidades en talleres de formación continua permitió cumplir con la gran misión encomendadas de educar con calidad y calidez.

\section{MÉTODO}

La investigación se realizó desde un enfoque cualitativo. Se orientó hacia un diseño de campo que permitió observar y recolectar información sobre el objeto de estudio en la institución elegida para el efecto. Posteriormente se realizó el análisis e interpretación de los resultados obtenidos, teniendo como punto de partida el distanciamiento por las causas conocidas, lo que permitió a través de la práctica del método colaborativo y con el apoyo de la tecnología acceder a un informe de las actividades realizadas por los docentes desde sus espacios y que se evidenciaron a través del entorno virtual utilizado.

El trabajo fue realizado de manera conjunta con el departamento de consejería estudiantil a fin de precautelar la información solicitada vía correo electrónico a los docentes. La población estuvo constituida por una población de 120 docentes de educación inicial, básica y bachillerato a los cuales se les consultó si deseaban participar o no en la investigación a través de una carta de consentimiento informado, la muestra la conformaron 120 docentes de todos los niveles educativos.

El instrumento utilizado para la recolección de la información fue la encuesta en un formulario enviado a los correos de los docentes, esta estuvo constituida por tres preguntas claves al directivo y a los docentes de la institución educativa, entre ellas ¿Conocer las experiencias, necesidades y deseos de cómo mejorar las habilidades sociales? Una vez obtenida la información se procedió a analizarla a través del sistema informática para obtener los porcentajes y estadística que nos ayudaron a establecer de forma jerárquica los requerimientos de los docentes para plantear una propuesta de solución.

\section{RESULTADOS Y DISCUSIÓN}

La realidad objetiva de los datos obtenidos mostró además la necesidad de implementar una propuesta de solución en función de mejorar el 
contexto social y de enseñanza de los docentes para alcanzar resultados efectivos en el logro de aprendizajes en los estudiantes. A continuación, se muestran los resultados en la Tabla 1, en la cual se puede apreciar que un $95 \%$ de docentes manifestaron que se sintieron agobiados frente a la modalidad online a pesar de tener muchos años de experiencia docente. Explicando que el sentimiento de opresión y angustia no lo soportaban por la presión del momento de pandemia y de los padres de familia.

Haciendo visible la necesidad de apoyarlos desde la institución educativa con estrategias para continuar el proceso de enseñanza con seguridad y armonía

Tabla 1. Actitud frente a la modalidad virtual.

\begin{tabular}{|c|c|c|c|c|c|}
\hline $\begin{array}{c}\text { Total de } \\
\text { docentes/120 }\end{array}$ & Niveles educativos & Agobiados & Desconcertados & Desconocimiento & Enfermos \\
\hline 15 & Navel initial & $60 \%$ & $17 \%$ & $10 \%$ & $13 \%$ \\
\hline 15 & Nivel preparatorio de educación básica & $68 \%$ & $15 \%$ & $12 \%$ & $5 \%$ \\
\hline 20 & Nivel media de educación básica & $80 \%$ & $10 \%$ & $6 \%$ & $4 \%$ \\
\hline 45 & Nivel superior de educación & $72 \%$ & $8 \%$ & $15 \%$ & $5 \%$ \\
\hline 25 & Bachillerato & $69 \%$ & $11 \%$ & $13 \%$ & $7 \%$ \\
\hline
\end{tabular}

De acuerdo con la descripción del estado social se observa que en todos los niveles educativo existía un alto porcentaje de docentes de educación básica, y superior donde se detecta que se sienten agobiados y en un menor porcentaje del nivel inicial desconcertados, y básica superior tenían desconocimiento de la modalidad, y un porcentaje importante del nivel inicial presentaron síntomas de enfermedad como niveles de depresión, agotamiento físico, dolores en los brazos, dificultades visuales , stress, inclusive empezaron a padecer de COVID 19, dejándose entrever que las medidas de aislamientos tomados por la expansión del virus para evitar tener riegos en la salud, no fueron suficientes y que estos comportamientos influían en el proceso de enseñanza de la modalidad virtual.
Para determinar que conflictos se les presentaron a los docentes en el proceso de la clase virtual, se presentan los resultados descritos en la Tabla 2, se evidenció que los docentes de educación básica y del nivel inicial en las salas virtuales los padres de los estudiantes de ese nivel fueron agresivos, de igual manera un menor porcentaje de docentes de educación superior básica manifestaron haber tenido padres agresivos, otro aspecto importante para los docentes es que la falta de conectividad se presentaba en mayor cantidad en estudiantes de la básica superior y bachillerato, los docentes manifestaron en $23 \%$ los estudiantes y padres del nivel preparatoria desconocían la metodología de educación virtual. 
Tabla 2. Conflictos con padres de familia y estudiantes.

\begin{tabular}{lcccc}
\hline \multicolumn{1}{c}{ Niveles educativos } & Padres agresivos & $\begin{array}{c}\text { No saber manejar } \\
\text { los conflictos }\end{array}$ & $\begin{array}{c}\text { Falta de } \\
\text { conectividad }\end{array}$ & $\begin{array}{c}\text { Descontento con } \\
\text { la Metodología }\end{array}$ \\
\hline Nivel inicial & $50 \%$ & $15 \%$ & $13 \%$ & $22 \%$ \\
Nivel preparatorio de educación básica & $40 \%$ & $25 \%$ & $12 \%$ & $23 \%$ \\
Nivel media de educación básica & $65 \%$ & $10 \%$ & $12 \%$ & $21 \%$ \\
Nivel superior de educación básica & $15 \%$ & $40 \%$ & $24 \%$ & $24 \%$ \\
Bachillerato & $25 \%$ & $35 \%$ & $20 \%$ & $20 \%$ \\
\hline
\end{tabular}

La variedad de respuestas obtenidas daba la idea de cuán difícil era ir moldeando la aplicación de la modalidad, bajo esos paramentos de conflictividad los docentes no tenían formas de hacer entender a los padres que todo fue de improvisación y acoplamiento y que poco a poco se iba a ir mejorando los procesos.

Los docentes desde sus representaciones expresaron que comprendían que eran tiempos difíciles y que debían ir mejorando los procesos de enseñanza virtual. Además, expresaron que la falta de incorporación de una conciencia plena en rutinas diarias de los padres les impedía adquirir habilidades saludables para enfrentar la situación de enojo de los padres en las salas virtuales. Dicho enfado obedece a que consideraban que no entendían las actividades escolares que se impartían a los estudiantes, además de la imposibilidad de seguirlos acompañando en la metodología se hacía presente en los reclamos a través de los medios comunicacionales que poseía la institución educativa.

En la Tabla 3 se evidencia las faltas de habilidades sociales que tienen los docentes en su proceso de enseñanza virtual. El mayor porcentaje que corresponde a los padres de bachillerato, se reflejó que los docentes de nivel inicial a básica superior los docentes indicaban que frente a los conflictos de los padres no sabían ¿cómo hacer peticiones? sobre actividades escolares incumplidas por los estudiantes o materiales que necesitaban porque todo les molestaba. Los docentes también manifestaron el descontrol que sentía frente a la falta de manejo de expresión de enfado o disconformidad por las situaciones que pasaban frente a los padres y estudiantes. 
Tabla 3. Habilidades sociales que necesitan desarrollar los docentes.

\begin{tabular}{lcccc}
\hline \multicolumn{1}{c}{ Niveles educativos } & $\begin{array}{c}\text { Falta de } \\
\text { autoexpresión de } \\
\text { situaciones } \\
\text { sociales }\end{array}$ & $\begin{array}{c}\text { No saber ecómo } \\
\text { hacer } \\
\text { peticiones? }\end{array}$ & $\begin{array}{c}\text { Falta de manejo } \\
\text { de expresión } \\
\text { de enfado o } \\
\text { disconformidadd }\end{array}$ & $\begin{array}{c}\text { Me cuesta } \\
\text { expresar } \\
\text { mi opinión en } \\
\text { grupos (clase, } \\
\text { reunión }\end{array}$ \\
\hline Nivel inicial & $30 \%$ & $35 \%$ & $13 \%$ & $22 \%$ \\
Nivel preparatorio de educación básica & $20 \%$ & $30 \%$ & $20 \%$ & $30 \%$ \\
Nivel media de educación básica & $25 \%$ & $35 \%$ & $14 \%$ & $26 \%$ \\
Nivel superior de educación básica & $23 \%$ & $30 \%$ & $20 \%$ & $27 \%$ \\
Bachillerato & $65 \%$ & $10 \%$ & $12 \%$ & $13 \%$ \\
\hline
\end{tabular}

Por otro lado, la falta de conocimiento de los docentes de manejar conflictos que quedaron evidenciadas en las grabaciones de las clases virtuales, conllevaron a sentir impotencia en los docentes y afectación de los procesos de socialización de la enseñanza con los estudiantes, que era evidenciado por los padres, impidiendo que los docentes realizaran sugerencias y opiniones frente a los conflictos escolares porque sentían temor de las acciones de los padres. Se consideró que los docentes necesitaban adquirir y fortalecer la forma de relacionarse con los otros a fin de gozar de un estado de bienestar para sentirse valorados y respetados.

\section{Discusión}

En relación a todos los antecedentes descritos y narrados en el marco de la temática estudiada, se explica a continuación que entre los resultados obtenidos destacan que un 98 $\%$ en los docentes la falta de autoexpresión de situaciones sociales, hacían que los docentes ante las agresiones que recibían les impedía manejar luego de que pasaba la situación volver a tener empatía con los padres. También se demostró que en los otros niveles de docentes de los niveles de inicial a básica superior, también tenían la misma problemática condicen por que los padres y estudiantes eran más susceptibles al cambio.

Una media entre el 30\% y $35 \%$ de docentes de educación media, básica y superior los se evidencia la problemática de $95 \%$ no saber cómo nacer peticiones para evitar que los padres dejen de interferir en la clase para que esta continúe y no se inicie conflictos de aprendizaje con los niños es la mayor preocupación de este nivel. A pesar que lo solicitan los padres no comprenden y después hacen comentarios negativos a las autoridades.

El porcentaje más preocupante es el $20 \% y$ $23 \%$ de docentes de educación comenta que siente la falta de manejo situacional frente a la expresión de enfado o inconformidad, de igual manera en los otros niveles $20 \%$ se destaca que en los niveles iniciales este porcentaje disminuye, de pronto porque son padres nuevos y se están adaptando a la modalidad educativa. No así en el nivel de bachillerato porque son muy pocos los padres que acompañan en proceso educativo virtual.

Se evidencia que un $30 \%$ de docentes del nivel de preparatoria les cuesta expresar su opinión en grupos (clase, reunión). Manifiestan que ellos siguen órdenes y que las mismas la 
explican para evitar contrariedades con los padres, tienen temor que los padres expresen su inconformidad y reclamos, y, en consecuencia, esto conlleve a que sufran algún problema laboral.

Toda la problemática presentada $\mathrm{y}$ concienciada desde los docentes hacen repensar en ¿cómo se está llevando a cabo la modalidad de enseñanza virtual?, habiendo planeado los docentes de manera general, que se debía hacer un cambio de estrategias a fin de concluir el ciclo académico con el éxito deseado. Para esto como investigadoras se plantea la propuesta de talleres de habilidades sociales, se realizó a través de 15 sesiones de capacitación virtuales, en la cual se llevaron a cabo trabajos colaborativos, en espacios de reflexión que les permitieron adquirir estrategias para dar solución a los conflictos que se les presentaban a los docentes. (Figura 1).

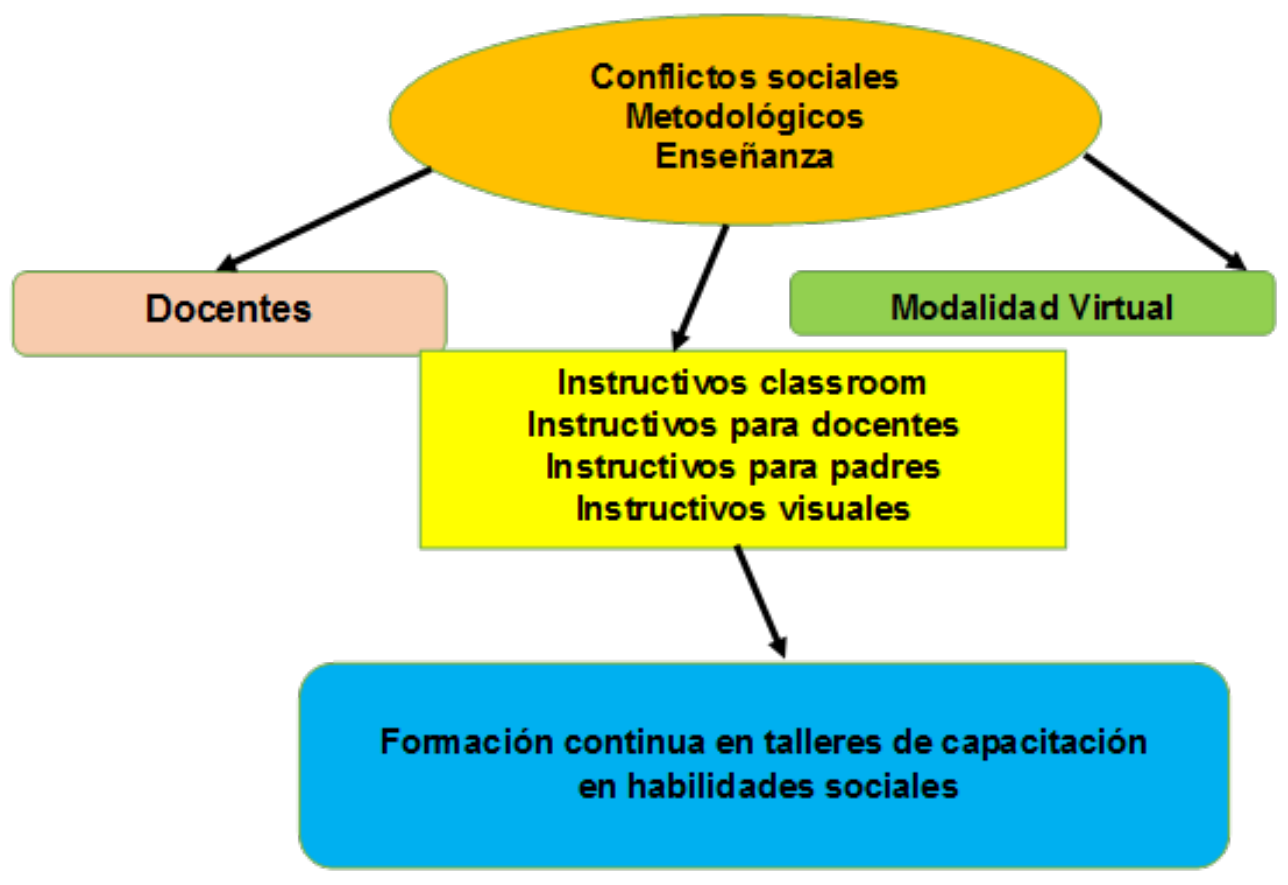

Figura 1. Propuesta de talleres de habilidades sociales.

\section{CONCLUSIONES}

La problemática más notable que los docentes con déficits en la adquisición de habilidades sociales es la barrera socioemocional que adquieren cuando se sienten acosados verbalmente por las personas que conforman su comunidad educativa. Toda propuesta para la adquisición de habilidades sociales incluye previamente una evaluación individualizada desde las experiencias de cada uno de los docentes que permitan conocer de forma real sus necesidades y dificultades, así como las habilidades concretas que necesitan desarrollar.

En cuanto a que las instituciones educativas deberían brindar espacios de reflexión a los docentes, no como informes académicos, sino más bien desde la percepción emocional y social para incentivarlos a afrontar las dificultades frente al proceso de enseñanza en la modalidad virtual. 
De los resultados expuestos en la investigación se considera que las habilidades sociales en los docentes, fomentan la comunicación asertiva entre los estudiantes y padres de familia, promocionando entre ellos estados de bienestar para sentirse valorados, y respetados de forma conjunta.

\section{REFERENCIAS}

Caballo, V. (1991). Habilidades sociales: definición y delimitación del concepto. https:// institutosalamanca.com/blog/habilidadessociales-definicion-delimitacion/

Cervera (2015) Educación y tecnología: nuevos escenarios de aprendizaje desde una visión transformadora. RUSC. Universitos and Knowledge Society Journal,12(2), 1-14
Gil, A. y León, R. (2019). El uso de las redes sociales entre los estudiantes de grado de maestro en un entorno exclusivamente online. Vivat Academia, (147), 23-40

Moscovici, S. (1979). La representación social: un concepto perdido. El Psicoanálisis, su imagen y su público, 2, 27-44

Vergara Quintero, M. (2008). Obtenido de http://biblioteca.clacso.edu.ar/Colombia/ alianza-cindeumz/20130801104940/ ArtMariadelCarmenVergara.pdf

Villarroel, G. E. (1999). Fermentum. Revista Venezolana de Sociología y Antropología

Villasana, N., y Dorrego, E. (2017). Habilidades sociales en entornos virtuales de trabajo colaborativo. https://www.redalyc.org/ pdf/3314/331427207003.pdf 